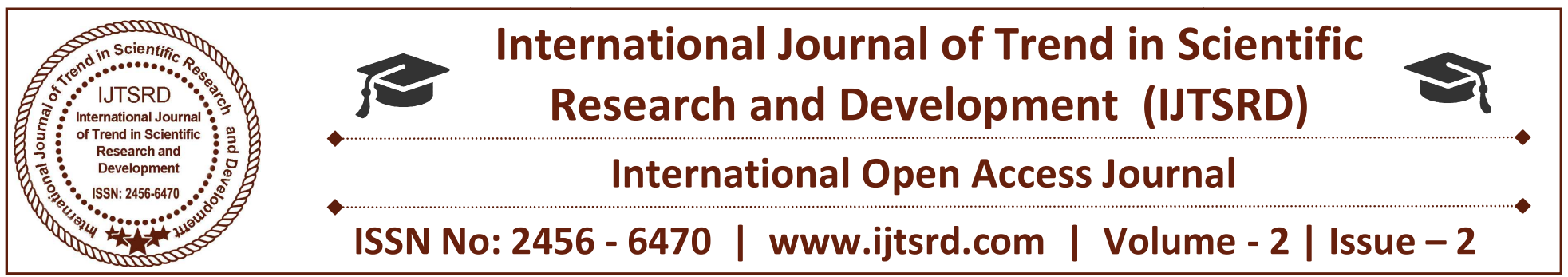

\title{
Enhanced RZ-Leach using Swarm Intelligence Technique
}

\author{
Pallavi Sharma \\ Computer Science and \\ Engineering, DAV Institute \\ of Engg \& Technology, \\ Jalandhar, India
}

\author{
Rajesh Kochher \\ Information Technology, DAV \\ Institute of Engg \& Technology, \\ Jalandhar, India
}

\author{
Varsha \\ Information Technology, CT \\ Group of Institutions, Shahpur, \\ Jalandhar, India
}

\section{ABSTRACT}

This paper elaborates the hybrid ACO-PSO technique based on the swarm intelligence. In this technique, basically the ant colony optimization is used to construct the relevant solution and several algorithm steps are set out and particle swarm optimization is used to optimize the parameters produced by ACO and these parameters can be selected self-adaptively. With these steps, the performance of algorithm enhanced in terms of energy consumption and the results prove the truism of the respective algorithm.

\section{Keywords: ACO (Ant Colony Optimization), PSO} (Particle Swarm Optimization), energy consumption.

\section{Introduction}

In the wireless sensor network, the sensor nodes are randomly deployed in the environment like civilian and military applications. Sensor nodes have limited battery life and cannot survive for longer time which is the main drawback of energy saving and this parameter is necessary for the enhancement of network. In wireless sensor network, the main components of sensors are their sensing unit, processing unit, communication unit and a power unit. In the sensing component, it senses the parameters and produces its analog signals. The task of processing unit is to process the signal and to control the sensor node. On the other hand, the communication component is used to send and receive the required data over several communication channels via short range radio. The power component consists of battery and positioning system of sensors.
Basically in the environment of wireless sensor network, sensor nodes $[5,10,12]$ are randomly deployed and one base station which is also called sink is situated in the region of examination. All the nodes send their data to the base station by using single hop communication and multi hop communication. Base station is also act as a gateway to internet i.e. gathering of data and transmission of information to client with the help of internet is finished by sink.

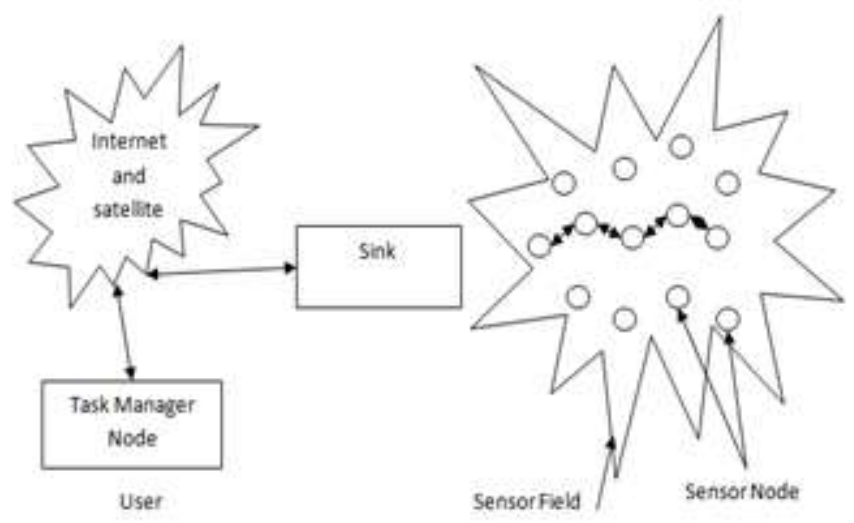

Fig.1. Wireless sensor network communication architecture

\section{Related Work}

WSNs consist of several sensor nodes and sink or base station which is placed in the required environment. The sensor nodes sense the data and send it to the base station. The processing of data is done at the base station. The base station can also send the commands to the sensor nodes. In the earlier time, data was send by single hop transmission from 
the sensor node to the base station. Transmission of data through the longer distance is not effective in lieu of consumption of energy. If distance is reduced then the energy will be save which enhances the network lifetime. So, to reduce the concept of single hop transmission, the solution was to adapt the concept of multi-hop routing. In multi-hop routing, the other nodes may participate and send data to the base station thereby reducing the distance of transmission and energy will be save. The drawback of multi-hop is that energy of nodes which is closer to the base station will drops quickly. To overcome this drawback, clustering is the solution. In clustering, the members of cluster can send their data to cluster head and then cluster head $[4,7]$ will transmit it to the base station. Whenever the internal nodes send their data to the cluster head called it as a intra cluster communication and when nodes send data to outside called it as a inter cluster communication.
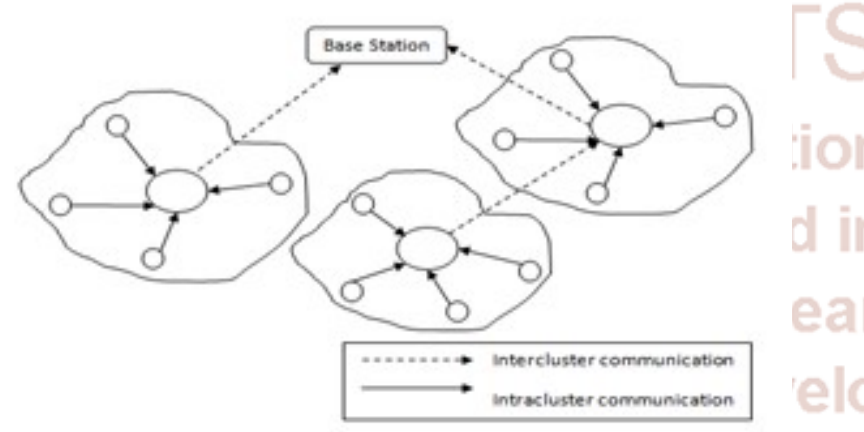

Fig.2. Data communication in a clustered network

$\mathrm{LEACH}$ is an effective adaptive clustering protocol $[1,6]$ and consists of two phases i.e. Set up phase and steady phase.

In the setup phase, the main issue is to make the cluster and formation of cluster head is also based on several parameters like energy, distance etc. On the other hand, in the steady phase, aggregate data from the normal node to the cluster head and then cluster head to the base station. Basically the steady phase is longer than that of setup phase because data aggregation takes more time. The first phase of the leach is the set up phase and it has three steps.

1) Cluster head advertisement

2) Set up of clusters

3) Creation of transmission schedule

In cluster head advertisement, the cluster head sends the information to all nodes that they become a cluster head on the basis of following formula,

$$
\operatorname{Th}(n d)=\left\{\begin{array}{cl}
\frac{p r}{1-p r *\left(\operatorname{rou} \bmod \frac{1}{p r}\right)} & , n d \in G r \\
0 & \text { otherwise }
\end{array}\right.
$$

The node becomes cluster head for that current round, if number is less than that of threshold value. In second step, nodes send joint request to cluster head and tell they all are members of respective cluster head. In the third step, selected cluster head make a transmission schedule for the nodes of their cluster. TDMA schedule is made and each node has to be send data in its limited time. In steady phase, cluster head send their data to the base station.

\section{Mobile Sink}

Mobile sink (MS) is a technique which helps in to minimize the energy consumption. The movement of MS[2,8,9] is inside or outside. It is of two types i.e. controlled manner or uncontrolled manner. If the location of mobile sink is in within area called controlled movement and if mobile sink moves randomly in the area then it is called uncontrolled movement. If sink is closer to the node then the distance is decreased which enhances the energy efficiency and network lifetime.

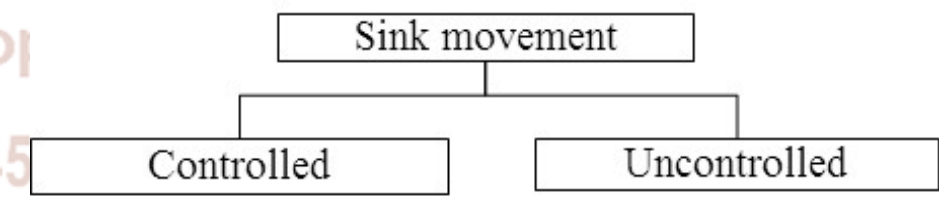

\section{Fig.3. Movement of Sink}

The hybrid ACO/PSO optimization technique has been applied to find out the shortest route to transmit the data, which may results into reduction of transmission distance and enhances the network lifetime.

\section{System Model}

In the system model, the sensor nodes are randomly deployed .Initial energy given to all the nodes is assumed to be $e_{o}$. Mobile sink moves on the $y$ axis and having unlimited energy. After each round the position of sink can be determined. The current system is made up of two parts i.e. set up phase and steady phase. The setup phase is having 3 phases. In the first phase, task ordination (TO) selection of cluster head and rendezvous nodes is selected. In the second phase, the cluster setup is formed, means 
clusters are organized. After formation of cluster, the last phase is scheduling in which a schedule is broadcast from cluster head to its members. So that transmission can be done according to the given time. In steady phase, transmission is done from node to mobile sink.

\section{A. Task ordination}

In the task ordination phase, node has to be decide whether they want to be rendezvous node then that particular node has send prerequisite. To become rendezvous node [3], the node has to be satisfy the condition. The distance of node has been compared with the mobile sink area. If node fulfills the condition then rendezvous node is labeled to them. The condition is following.

$$
\frac{\mathrm{y}_{\mathrm{w}}}{2}(1+\mathrm{Rx})<=\mathrm{y}_{\mathrm{y}}<=\frac{\mathrm{y}_{\mathrm{w}}}{2}(1-\mathrm{Rx})
$$

where $\mathrm{y}_{\mathrm{w}}$ represents the sampling region width, $\mathrm{y}_{\mathrm{y}}$ represents the position of node in $y$-direction and constant $\mathrm{R}_{\mathrm{x}}$ must have value $<1$.

\section{B. Cluster selection}

Once cluster head[11] and rendezvous node are selected then cluster head and rendezvous node broadcast the data to normal node using CSMA protocol. To hear the message broadcast by cluster head and rendezvous node, the receiver of normal node must be 'ON'. The normal nodes then decide their cluster and find rendezvous node closer to it. The decision has been according to the distance from cluster head and rendezvous nodes. If signal strength is more, then the distance will be less means normal node attaches to that cluster head having lesser distance.

\section{Data transmission}

To reduce the utilization of energy the next step is to find the shortest route for data transmission after the creation of set up has been done. From the swarm intelligence, the concept of hybrid $\mathrm{ACO} / \mathrm{PSO}$ is applied. First of all, Ant Colony Optimization (ACO) technique has been applied to find the shortest route from node to the sink. The probability to get the optimized route is given by formula

$$
\operatorname{Pro}\left(C_{x, y} \mid S^{p r}\right)=\frac{\tau_{x, y^{\alpha} * \mathfrak{y}_{x, y} \beta}}{\sum_{x, y} \in \tau_{x, y^{\alpha}\left(S^{p r}\right)}{ }^{\eta_{x, y} \beta}}
$$

where, $S^{p r}$ is partial solution, $\mathrm{P}$ is set of all the paths from the city $\mathrm{x}$ to all adjacent cities still not visited by ant, $C_{x, y}$ is the path from the city x to y, Pro is the probability, $\tau_{x, y}$ is the amount of pheromone in the path $C_{x, y}, \mathfrak{y}_{x, y}$ is the heuristic factor usually $\mathfrak{y}_{x, y}=\frac{Q}{d_{x, y}}$ , where $d_{x, y}$ be the distance along the cities $\mathrm{x}$ and $\mathrm{y}, \mathrm{Q}$ be the some constant and ' $\alpha$ ' and ' $\beta$ ' are algorithm parameters

After getting the set of results of shortest routes, particle swarm optimization (PSO) is applied to get refine the results. It provides a population based search technique that uses personal experience (Pbest), overall experience (Gbest) and the current position of particle to find the coming location in the area of search. The PSO algorithm works as given below.

- Set parameters $\mathrm{w}_{\min }, \mathrm{w}_{\max }, \mathrm{C} 1$ and $\mathrm{C} 2$ (ranges from 2 to 2.05) of PSO.

- Initialize population of particle having population 'P' (usually varies from 10 to 100) and velocities 'Vel'(initial velocity is $10 \%$ of position).

- set iteration $i=1$

- calculate fitness of particle $F_{x}^{i}=f\left(P_{x}^{i}\right)$, find index of best particle $b$

- Select Pbest $_{x}^{i}=P_{x}^{i}$ and Gbest ${ }^{i}=P_{b}^{i}$

- $w=w_{\max }-i *\left(w_{\max }-w_{\min }\right) /$ Maxit where, Max it varies from 500 to 10000

- Update $\mathrm{V}$ and position of particle $V_{x, y}^{i+1}=\mathrm{w} * V_{x, y}^{i}+C 1 * \operatorname{rand}() *\left(\right.$ Pbest $\left._{x, y}^{i}-P_{x, y}^{i}\right)$ $\mathrm{V}^{i+1}{ }_{\mathrm{x}, \mathrm{y}}=\mathrm{w}^{*} \mathrm{~V}_{\mathrm{x}, \mathrm{y}}^{\mathrm{i}}+\mathrm{C} 1^{*} \operatorname{rand}()^{*}\left(\mathrm{Pbest}_{\mathrm{x}, \mathrm{y}}-\mathrm{P}_{\mathrm{x}, \mathrm{y}}^{\mathrm{i}}\right)+\mathrm{C} 2 *$ $\operatorname{rand}()^{*}\left(\right.$ Gbest $\left._{\mathrm{x}, \mathrm{y}}^{\mathrm{i}}-\mathrm{P}_{\mathrm{x}, \mathrm{y}}^{\mathrm{i}}\right)$

$P_{x, y}^{i+1}=P_{x, y}^{i}+V_{x, y}^{i+1}$

- Evaluate fitness $F_{x}^{i+1}=f\left(P_{x}^{i+1}\right)$, find index of best particle b1

- Update Pbest if $F_{x}^{i+1}<F_{x}^{i}$ Then Pbest $t_{x}^{i+1}=$ $P_{x}^{i+1}$ else Pbest $_{x}^{i+1}=$ Pbest $_{x}^{i}$

- Update Gbest if $F_{b 1}^{i+1}<F_{b}^{i}$ Then Gbest $^{i+1}=$ Pbest $^{i+1}, \mathrm{~b}=\mathrm{b} 1$, else Gbest $^{i+1}=$ Gbest $^{i}$

- If $i<$ Maxit then $i=i+1$ and goto step 6 else goto step 12

- $\quad$ Print Gbest ${ }^{i}$ 


\section{Simulation}

In this simulation environment, the 100 sensor nodes are deployed in the area of $(100,100)$. The MATLAB simulator is used for the given experiment. The parameters are listed below in the given table. The metrics used for the simulation are:-

- Number of dead nodes

- Number of alive nodes

- Number of packets send to base station

- Remaining Energy

\section{Table1:- Simulation Parameters}

\begin{tabular}{|l|l|}
\hline Parameters & Value \\
\hline Area $(\mathrm{x}, \mathrm{y})$ & $\begin{array}{l}150 * 150,200 * 200, \\
250 * 250\end{array}$ \\
\hline Base Station(x,y) & Moving \\
\hline Number of nodes & 100 \\
\hline Probability & 0.1 \\
\hline Initial Energy & $0.5 \mathrm{~J}$ \\
\hline Transmitter Energy & $50 \mathrm{~nJ} / \mathrm{bit}$ \\
\hline Receiver Energy & $50 \mathrm{~nJ} / \mathrm{bit} / \mathrm{nter} / \mathrm{hat}$ \\
\hline $\begin{array}{l}\text { Free } \\
\text { Energy(amplifier) }\end{array}$ & $1.0 \mathrm{~nJ} / \mathrm{bit} / \mathrm{m}^{\wedge} 2$ \\
\hline Multipath Energy & $0.0013 \mathrm{~nJ} / \mathrm{bit} / \mathrm{m}^{\wedge} 2 \mathrm{~s}$ \\
\hline Number of rounds & 10,000 \\
\hline Message Size & $4000 \mathrm{bits}$ \\
\hline
\end{tabular}

This is the simulation environment of wsn in which 100 nodes are deployed and position of sink is moving along y axis.

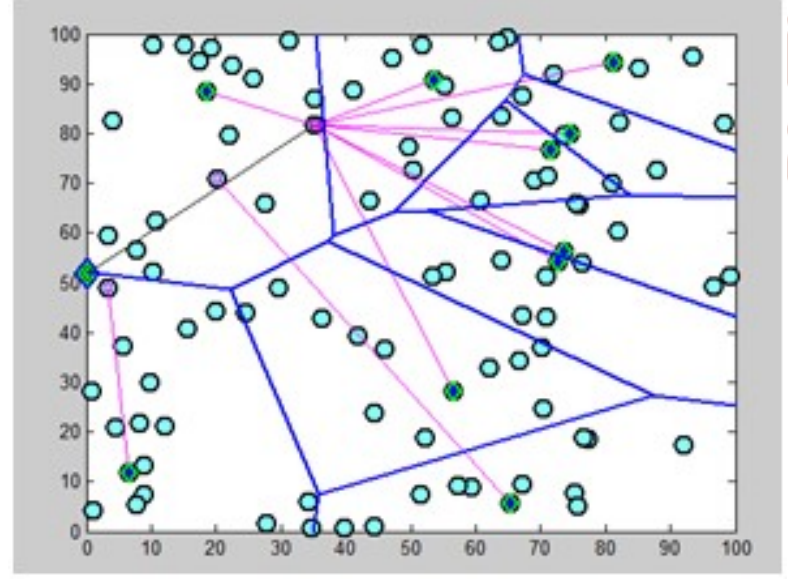

Fig.4. Square region of $100 * 100$ having 100 nodes
Figure 2 shows a network of $100 * 100 \mathrm{~m}$ having 100 nodes.

While considering network with 100 nodes we compare the performance of proposed approach for 3 regional sizes i.e. $150 \mathrm{~m} * 150 \mathrm{~m}, 250 \mathrm{~m} * 250 \mathrm{~m}$ and $350 \mathrm{~m} * 350 \mathrm{~m}$, against number of rounds.

Case 1:- On the area of $150 * 150 \mathrm{~m}$

Dead Nodes:- This is the graph of dead nodes in RZLEACH and ACO/PSORZLEACH protocol.The network lifetime can be evaluated by using the number of dead nodes. It has been found that the number of nodes die earlier in RZLEACH protocol. Here, we can see from the graph that all the nodes are die at the round of 500 in case of RZLEACH and 750 in case of ACO/PSORZLEACH.

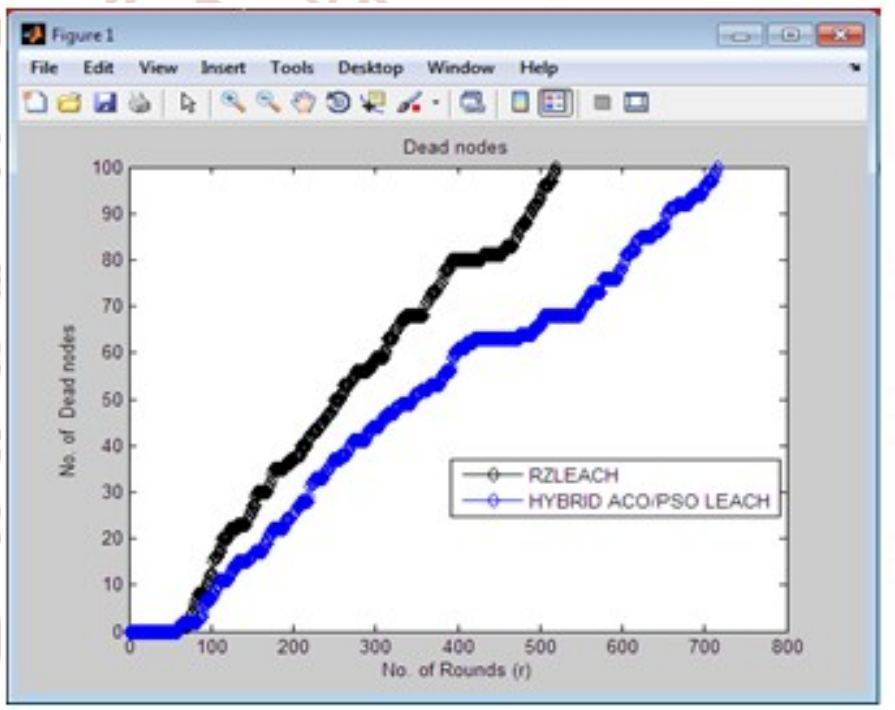

Fig 5: Dead nodes Vs Rounds

Alive Nodes:- This is the graph of alive nodes in RZLEACH and ACO/PSORZLEACH protocol. It has been found that the number of nodes alive much more in ACO/PSORZLEACH protocol. Here, we can see from the graph that the nodes are alive at the round of 510 in case of RZLEACH and 710 in case of ACO/PSORZLEACH. 


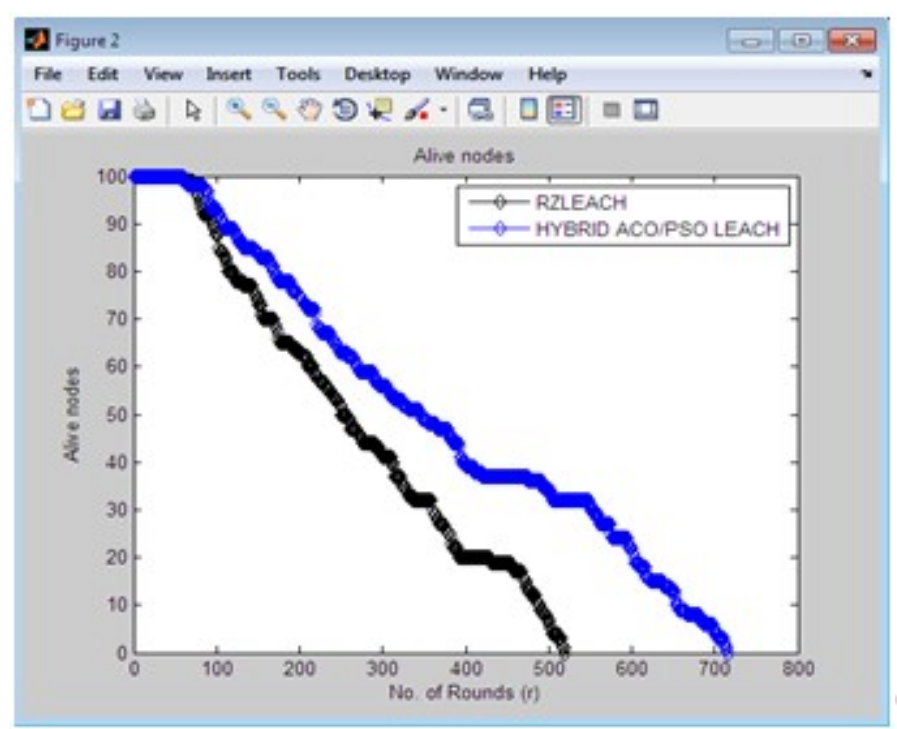

Fig 6: Alive nodes Vs Rounds

\section{Packets Send to base station:-}

This is the graph of Packet send to base station after simulation. This graph shows the total number of packets send to the base station by the sensor nodes. At the round of 520, the total number of packets send to base station is 2000 in the case of RZLEACH protocol and in case of PSORZLEACH, the packets send to base station is 2800 .

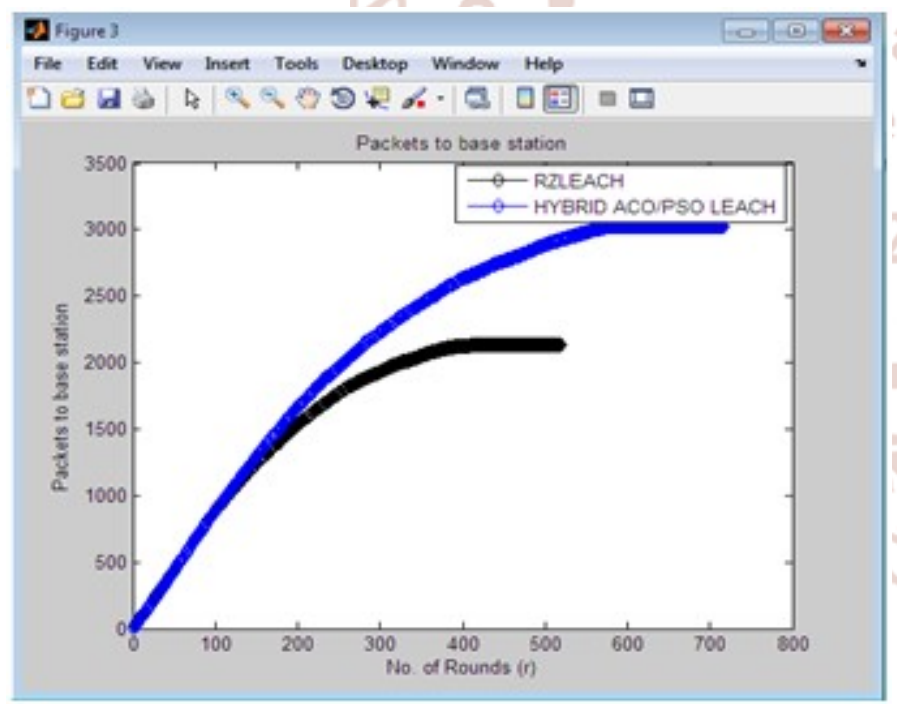

Fig 7: packet send to BS Vs Rounds

\section{Remaining Energy:-}

This is the graph of remaining energy, how much energy is left with the rounds. From the graph, we can see the remaining energy with RZLEACH goes to 500 rounds, whereas in the case of ACO/PSORZLEACH the remaining energy goes to 700 rounds means more work can be done with ACO/PSORZLEACH protocol.

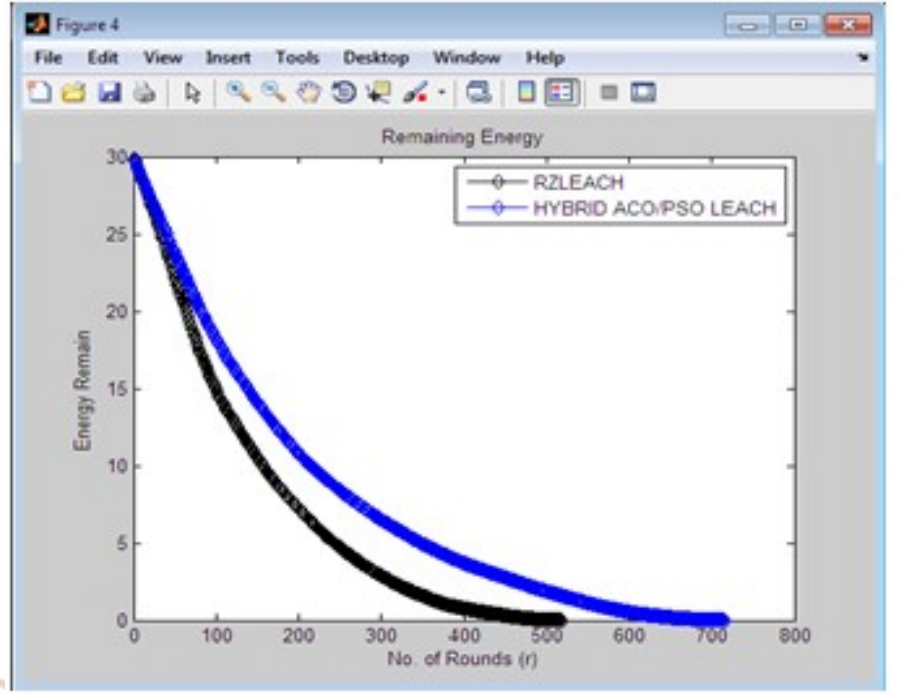

Fig 8: Remaining Energy Vs Rounds

Case2: On the area of $200 * 200 \mathrm{~m}$

Dead Nodes:- This is the graph of dead nodes in RZLEACH and ACO/PSORZLEACH protocol. The network lifetime can be evaluated by using the number of dead nodes. It has been found that the number of nodes die earlier in RZLEACH protocol. Here, we can see from the graph that all the nodes are die at the round of 520 in case of RZLEACH and 680 in case of ACO/PSORZLEACH.

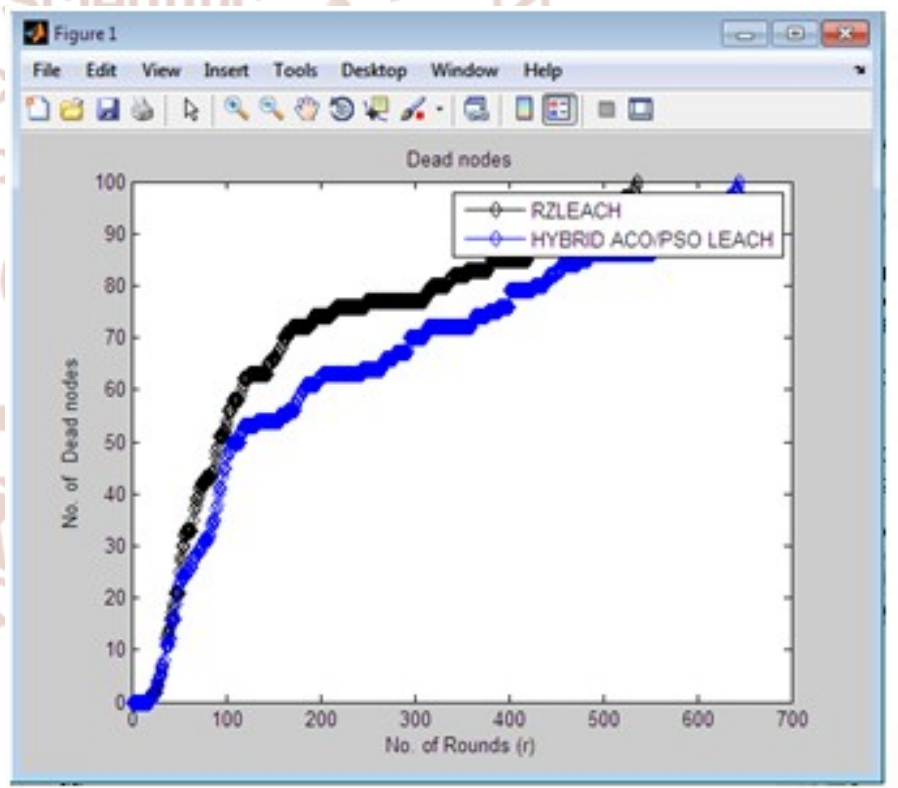

Fig 9: Dead nodes Vs Rounds

Alive Nodes:- This is the graph of alive nodes in RZLEACH and ACO/PSORZLEACH protocol. It has been found that the number of nodes alive much more in ACO/PSORZLEACH protocol. Here, we can see from the graph that the nodes are alive at the round of 540 in case of RZLEACH and 650 in case of ACO/PSORZLEACH. 


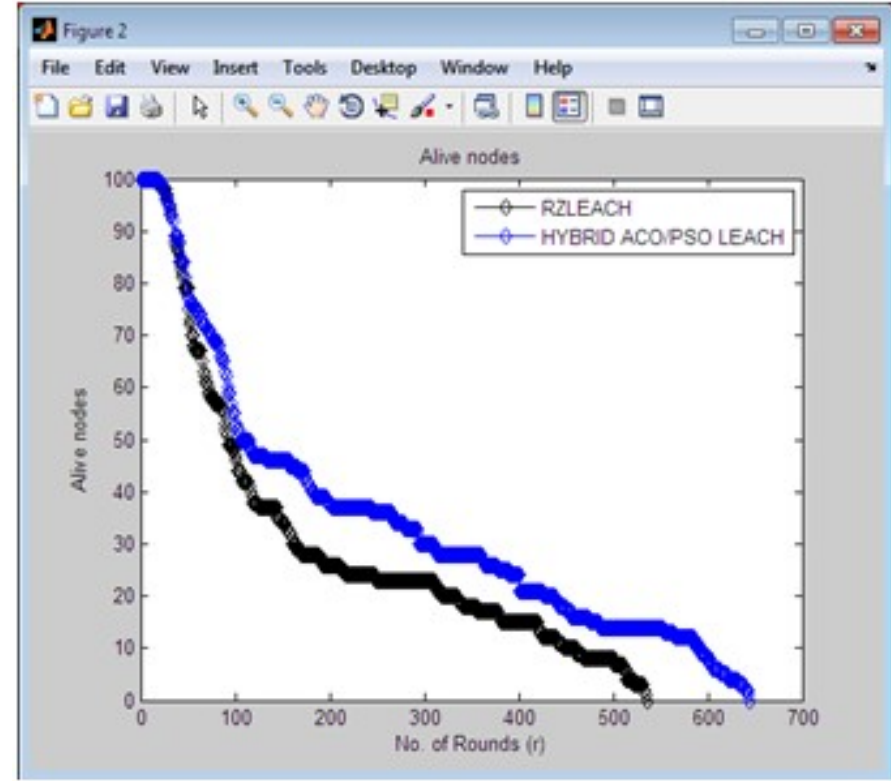

Fig 10: Alive nodes Vs Rounds

work can be done with ACO/PSORZLEACH protocol.

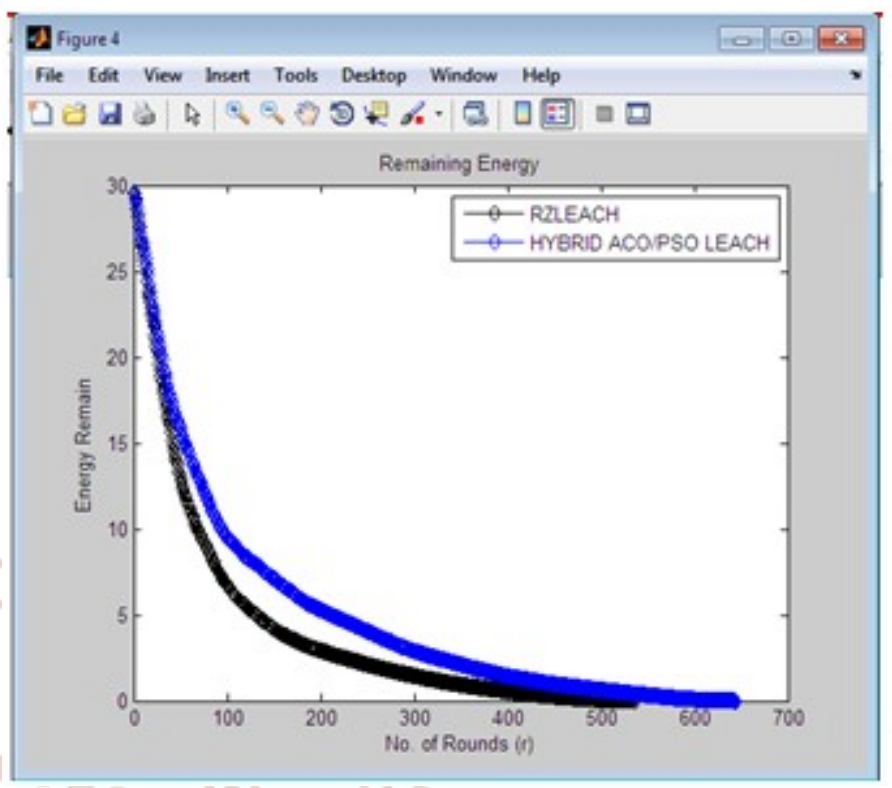

\section{Packets Send to base station:-}

This is the graph of Packet send to base station after simulation. This graph shows the total number of packets send to the base station by the sensor nodes. At the round of 540, the total number of packets send to base station is 650 in the case of RZLEACH protocol and in case of PSORZLEACH, the packets send to base station is 1000 .

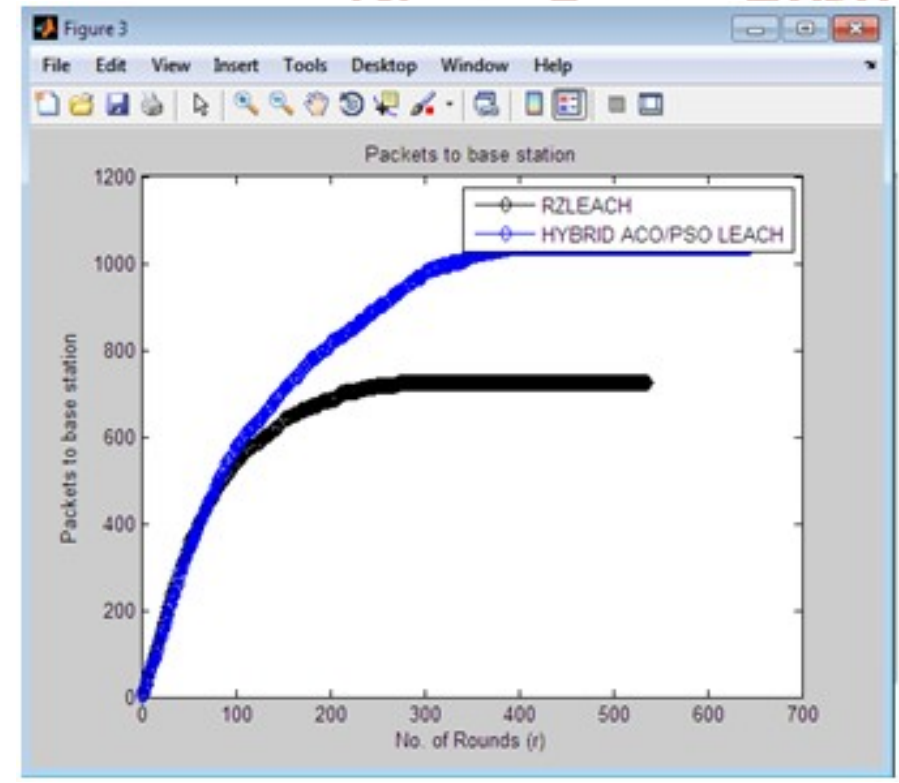

Fig 11: Packet send to BS Vs Rounds

\section{Remaining Energy:-}

This is the graph of remaining energy, how much energy is left with the rounds. From the graph, we can see the remaining energy with RZLEACH goes to 510 rounds, whereas in the case of ACO/PSORZLEACH the remaining energy goes to 620 rounds means more

Fig 12: Remaining Energy Vs Rounds

Case3: On the area of $250 * 250 \mathrm{~m}$

Dead Nodes:- This is the graph of dead nodes in RZLEACH and ACO/PSORZLEACH protocol.The network lifetime can be evaluated by using the number of dead nodes. It has been found that the number of nodes die earlier in RZLEACH protocol. Here, we can see from the graph that all the nodes are die at the round of 510 in case of RZLEACH and 550 in case of $\mathrm{ACO} / \mathrm{PSORZLEACH}$.

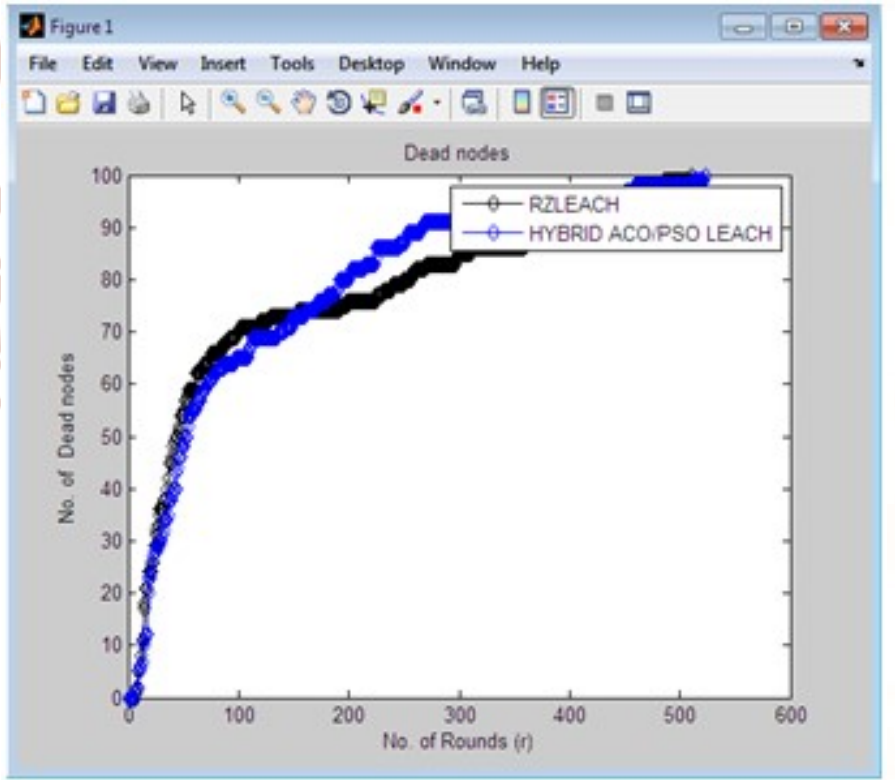

Fig 13: Dead nodes Vs Rounds

Alive Nodes:- This is the graph of alive nodes in RZLEACH and ACO/PSORZLEACH protocol. It has been found that the number of nodes alive much more in ACO/PSORZLEACH protocol. Here, we can see 
from the graph that the nodes are alive at the round of 500 in case of RZLEACH and 510 in case of ACO/PSORZLEACH.

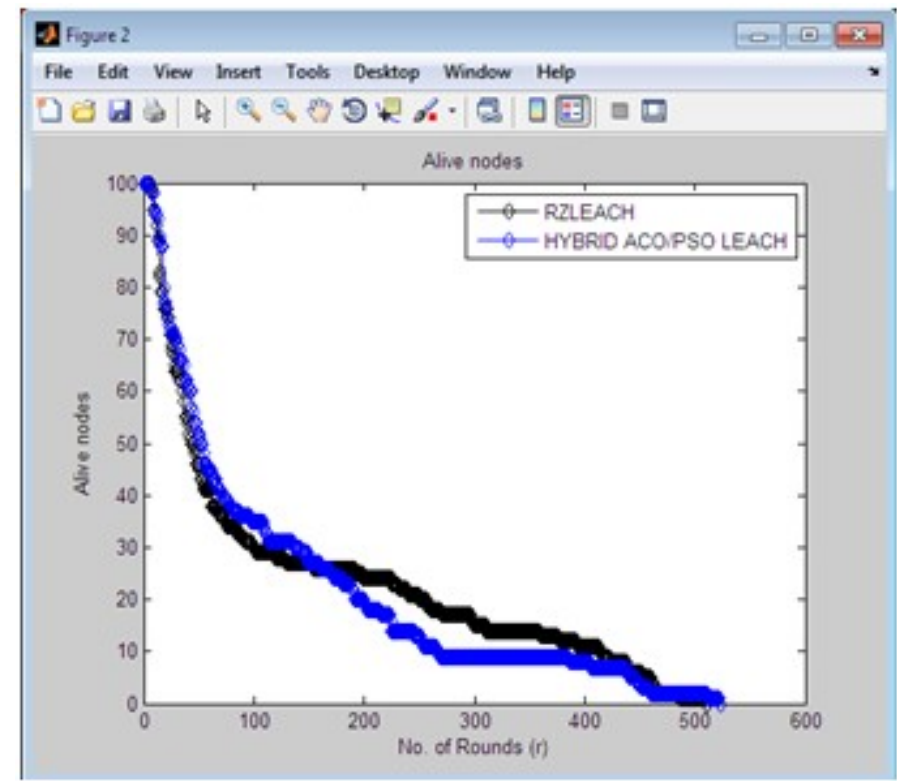

Fig 14: Alive nodes Vs Rounds

\section{Packets Send to base station:-}

This is the graph of Packet send to base station after simulation. This graph shows the total number of packets send to the base station by the sensor nodes. At the round of 520, the total number of packets send to base station is 300 in the case of RZLEACH protocol and in case of PSORZLEACH, the packets send to base station is 380 .

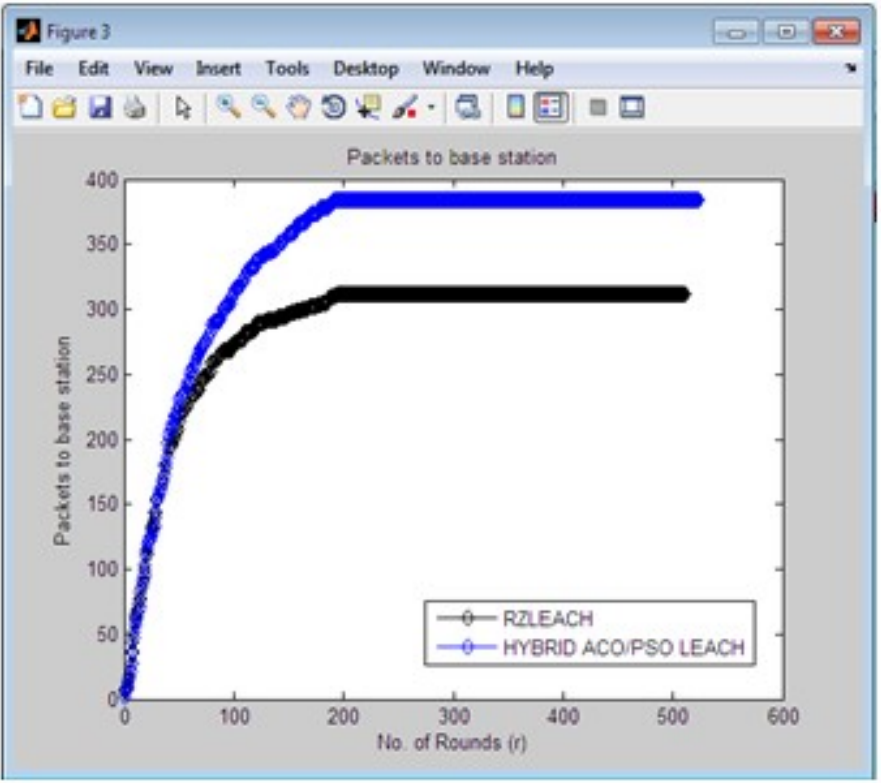

Fig 15: Packet send to BS Vs Rounds

\section{Remaining Energy:-}

This is the graph of remaining energy, how much energy is left with the rounds. From the graph, we can see the remaining energy with RZLEACH goes to 400 rounds, whereas in the case of ACO/PSORZLEACH the remaining energy goes to 510 rounds means more work can be done with ACO/PSORZLEACH protocol.

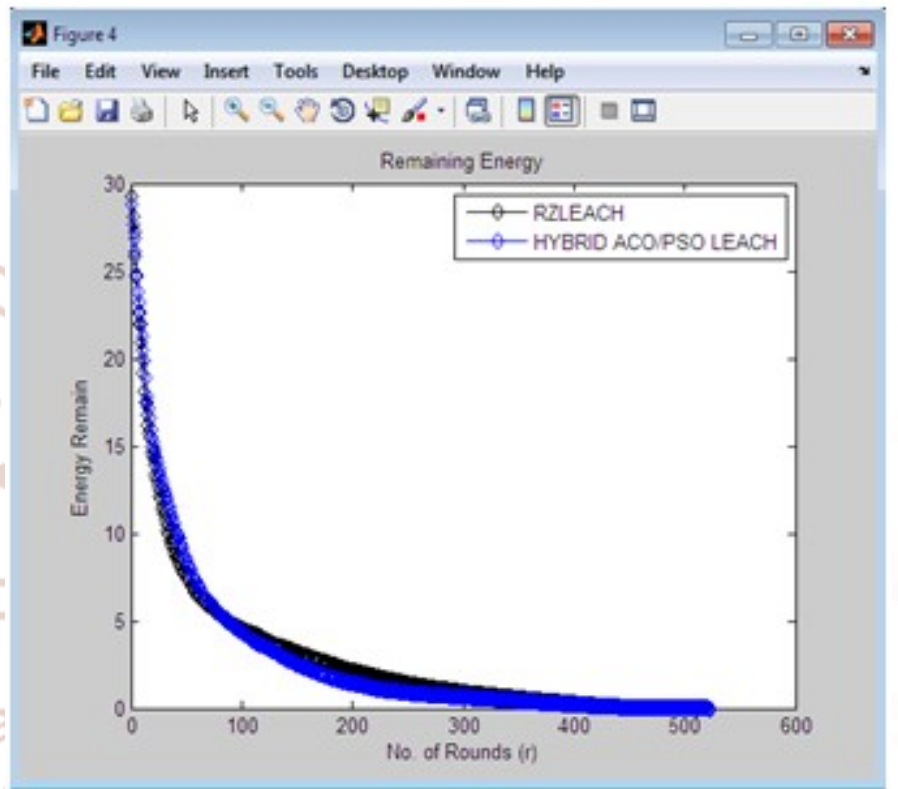

Fig 16: Remaining Energy Vs Rounds

The remaining energy is an important consideration for WSN, which is computed using

$\operatorname{RemEng}(r e)=\sum_{N=1}^{n} E_{r r}(N d)$

Where $E_{r r}(\mathrm{Nd})$ is the energy of Ndth node for $\mathrm{rr}^{\text {th }}$ round.

In all of the cases i.e. for case 1 and case 2 and case 3 , the proposed system performs better than RZ LEACH.

\section{CONCLUSION}

To minimize the energy consumption, we proposed a hybrid routing technique which is called hybrid ACO/PSO based on swarm intelligence. Hybrid $\mathrm{ACO} / \mathrm{PSO}$ helps to find out the shortest distance for routing process and thereby enhancement can be done in network lifetime. For the proposed work, we are using wireless communication and data analysis toolbox of Matlab 2013a. The proposed protocol shows the better improvement over existing protocol. But this work has not taken into account the utilization of 3D WSNs, which are becoming major 
area of research in these days. Therefore in near future work we will extend the planned technique for 3D WSNs environment.

\section{REFERENCES}

1. Mottaghi, Saeid, and Mohammad Reza Zahabi. "Optimizing LEACH clustering algorithm with mobile sink and rendezvous nodes." AEUInternational Journal of Electronics and Communications 69, no. 2: 507-514, 2015.

2. Yu Gu; Inf. Syst. Archit. Sci. Res. Div., Nat. Inst. of Inf ; Yusheng Ji;Jie Li;Baohua Zhao. "Efficient Scheduling for the Mobile Sink in Wireless Sensor Networks with Delay Constraint",IEEE Trans Parallel Distrib System;24(july(7)):pp 131020. IEEE Tokyo, Japan , 2013.

3. Konstantopoulos, C; Dept. of Inf., Univ. of Piraeus, Piraeus,; Pantziou, G.; Gavalas, Damianos; Mpitziopoulos, A. "A RendezvousBased Approach Enabling Energy-Efficient Sensory Data Collection with Mobile Sinks In:IEEE Trans Parallel Distrib System;23(may(5)):pp 809-17. IEEE, Greece ,2013.

4. Khalid Hussain, Abdul Hanan Abdullah, Khalid M. Awan, Faraz Ahsan and Akhtab Hussain," Cluster Head Election Schemes for WSN and MANET: A Survey", World Applied Sciences Journal 23 (5): 611-620, ISSN 1818-4952, 2013.

5. Wang Liu; Dept. of Comput. Sci., Univ. of Sci. \& Technol. of China, Hefei; Kejie Lu; Jianping Wang ; Guoliang Xing, "Performance Analysis of Wireless Sensor Networks With Mobile Sinks" IEEE Trans Veh Technol;61(July(6)):2777-88 IEEE, China , 2012.

6. Reetika Munjal, Bhavneesh Malik "Approach for Improvement in LEACH Protocol for Wireless Sensor Network", Second International Conference on Advanced Computing \& Communication Technologies (IEEE); pp.517521.IEEE, Rohtak, Haryana 7-8 Jan 2012.

7. Peng, J., Chengdong, W., Yunzhou, Z. and Fei, C., "A Low-Energy Adaptive Clustering Routing Protocol of Wireless Sensor Networks", IEEE International Conference on Wireless Communications, Networking and Mobile Computing (WiCOM), September, pp. 1-4. IEEE, 2011 .
8. Weifa Liang; Sch. of Comput. Sci., Australian Nat. Univ., Canberra, ACT, Australia ; Jun Luo; $\mathrm{Xu} \mathrm{Xu.} \mathrm{"Prolonging} \mathrm{Network} \mathrm{Lifetime} \mathrm{via} \mathrm{a}$ Controlled Mobile Sink in Wireless Sensor Networks" In: Global telecommunication conference (IEEE GLOBECOM 2010), pp.1-6. Australia, December 2010.

9. JaspinderKaur, Varsha "A New Approach for Energy Efficient Linear Cluster Handling Protocol In WSN "International journal of computer science and information security (ijcsis) March 2016, Vol. 14 No. 3 (Thomson Reuters).

10. Harshdeep, Varsha. "Tabu Search and Tree Based Energy Efficient Protocols for Wireless Sensor Networks". International Journal of Advanced Research in Computer Science and Software Engineering (IJARCSSE) ISSN: 2277-128X, Impact Factor: 2.5, Vol-5, Issue-9, Page no 923933, September2015.

11. Varsha Sahni, Manju Bala et al "TABU Search Based Cluster Head Selection in Stable Election Protocol" International Journal on Recent Trends in Computing and Communication, Volume: 4, Issue: 8 , pp: 90-94.

12. Isha, Varsha, "Study on Co-operative Communication for Energy Efficient Routing in Wireless Sensor Network", International Journal of Science and Research (IJSR), https://www.ijsr.net/archive/v5i8/v5i8.php, Volume 5 Issue 8, August 2016, $297-300$. 